\title{
Applications of a Derivative Function of One Variable
}

\author{
${ }^{1}$ Anton N. Karamyshev, ${ }^{2}$ Zhanna I. Zaytseva \\ ${ }^{1,2}$ Kazan Federal University \\ Email: antonkar2005@yandex.ru
}

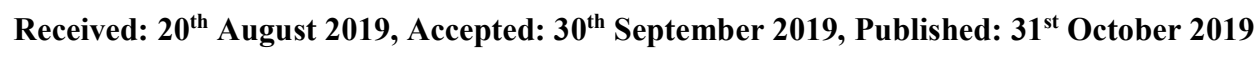

\begin{abstract}
The relevance of the topic of this article is derived from the education informatization process - the process of providing the education sector with the methodology and practice of the development and optimal use of modern computer technologies, and therefore - with the necessary changes in the content of education, its structure and the introduction of new computer technologies in the educational process. The purpose of this article is the methodology of using information technology in the educational process of teaching mathematics by creating and using new forms of software pedagogical products using advanced computer technology. The leading method for studying this problem is the methodological analysis and subsequent synthesis methods, which allows identifying the necessary ways and methods for creating a pedagogical program product by analyzing the didactic content of the section "Differential calculation of the function of one variable" of the university course in higher mathematics, as well as the instrumental capabilities of the Mathematica mathematical computer environment. The article describes the main aspects of the work of a mathematics teacher, who is not a specialist in programming, in compiling and using a simulator in the educational process on the topic "Application of the derivative function of one variable" using the Mathematica computer mathematical system. Article materials may be useful for the teachers of mathematical disciplines of higher educational institutions. The simulator on the topic "Application of the derivative function of one variable", which was created and intended for use in the Mathematica computer mathematical system, can serve as an example of the development of similar pedagogical software products.
\end{abstract}

\section{Keywords}

Simulator, Computer Mathematical System, Differential Calculation, Computerization of Mathematical Education, Information Technology.

\section{Introduction}

The application of differential calculation, in particular, the derivative function of one variable, is a powerful tool for solving applied tasks, as we know. The representatives of various specialties have to work with this type of tasks: process engineers organize production so that the enterprise can produce as many products as possible; designers are developing new devices for spacecraft with an optimal reduction in their mass; economists are trying to build more profitable transport links for the enterprises between the sources of raw materials and the production facilities, etc.

Here is a list of some practical problems that can be solved using the derivative:

1. It is necessary to make an open box of the greatest volume from a rectangular tin sheet measuring $25 \times 40 \mathrm{~cm}$. You need to cut square corners for this purpose. Depending on the cut square size, the boxes with different volumes are obtained. Therefore, it is important to calculate the side length of the cut squares so that the entire box has the largest volume. It is used the ability to find the extremum of a function of one variable to solve this task. $[3,4]$

2. The charge flowing through the conductor varies according to the law $q=\sin (2 t-10)$.Find the current strength at time $\mathrm{t}=5 \mathrm{sec}$. It is used the ability to find the value of the first derivative at a point to solve this task.

3. An electric heater consumes power from a current source, whose EDS is $3 \mathrm{~V}$ and internal resistance is $2 \mathrm{Ohms}$. What resistance should the device have to release maximum power? It is used the ability to find the extremum of a function of one variable to solve this task.

However, it should be noted that there has been a noticeable decrease in interest in mathematics on the part of both pupils and students, and society as a whole in recent years. Mathematics is perceived not as one of the means of professional activity, but as an abstract science, which does not come in handy in the future professional activity. The students do not want to spend time and effort to study such a complex, but interesting and exciting subject as mathematics. And therefore, in order to enhance the cognitive activity of students and develop their ability to independently solve complex problems, it is necessary to strengthen the motivation of students; it is impossible to achieve this without the introduction of new methods and technologies into the educational process. [6]

\section{Text of Article}

As an effective means of new information and communication technologies, we propose to use in the educational process a simulator on the topic "Application of the derivative function of one variable", which was developed by the authors in the Mathematica computer mathematical system. This simulator has all the properties of a training-controlling program and is designed to organize the students' independent work (both classroom and home) when studying the section "Differential calculation of the function of one variable". This article does not provide the program code for compiling 
the simulator (it is protected by the copyright certificate [5] and described in [1], [2]); the didactic sequence of the simulator compilation by the teacher, the student's actions and the simulator's reaction on this topic are traced here.

Let us consider the structure and operation of the simulator. Each option has a record: "Then select the hidden cell with the left mouse button, press Shift + Enter in turn and follow the further instructions". After this instruction has been fulfilled, a dialog box appears with the inscription what you need to write in it (Figure 1), and the simulator enters a dialogue with the user.

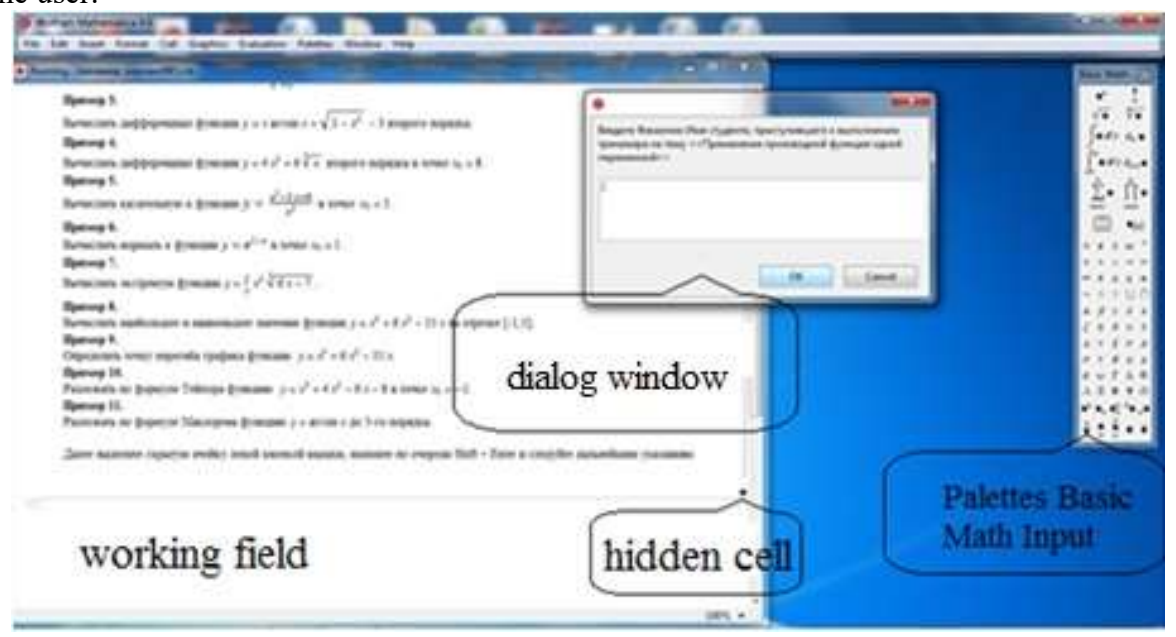

Fig. 1: General View of the Simulator

The following are entered in the dialog box: last name, first name of the student, group number and option number being executed, as well as the conditions of examples. The simulator controls each step of the student, making the necessary calculations in parallel with him/her and comparing them with those introduced by the student. If the student introduces another condition, the simulator will not specify an error, but this new condition will be reflected in the working field, which will allow the teacher finding the substitution. After introducing the example conditions, the student needs to solve it and write down the intermediate results and the received answer in the dialog box sequentially. All actions performed are reflected in the working field. If the entered result is correct, the entry "Well done! The number of errors is 0 " will appear in the working field, and the computer will offer proceeding with further instructions; otherwise, another entry will appear in the working field: "You made a mistake. You have 2 attempts left". In this case, it is possible to independently find an error in the example solution. It is necessary to resolve and write the corrected value in the dialog box; if it is correct, the entry "Well done! The number of errors is 1" will occur, and you can proceed to the implementation of further instructions. Otherwise there will be a record "You made a mistake. You have 1 attempt left". And you can try again to find the error. In total, three errors can be made, after which the simulator displays the correct answer and the number of errors made. [7]

Let us take a closer look at the simulator on the topic "Application of the derivative function of one variable", which has been created in the Mathematica computer mathematical system and contains the following examples:

Example 1. Calculate function differential $y=f(x)$.

An example condition (function $f(x)$ ), a result of the intermediate solution (derivative of the function) and then the answer (differential of the function) are introduced.

In total, 6 errors can be made.

Example 2. Calculate function differential $y=f(x)$ at point $x_{0}$.

An example condition (function $f(x)$, point $x_{0}$ ), a result of the intermediate solution (differential of the function) and then the answer (value of the function differential at the point $x_{0}$ ) are introduced.

In total, 6 errors can be made.

Example 3. Calculate the second-order function differential $y=f(x)$.

An example condition (function $f(x)$ ), results of the intermediate solution (derivative of the first-order function, derivative of the second-order function) and then the answer (differential of the second-order function) are introduced. In total, 9 errors can be made.

Example 4. Calculate the second-order function differential $y=f(x)$ at the point $x_{0}$. 
An example condition (function $f(x)$, point $x_{0}$ ), results of the intermediate solution (derivative of a first-order function, derivative of a second-order function) and then the answer (differential value of a second-order function at a point $\left.x_{0}\right)$ are introduced.

In total, 9 errors can be made.

Example 5. Find the tangent equation to the graph of the function $y=f(x)$ at the point $x_{0}$.

An example condition (function $f(x)$, point $x_{0}$ ), results of the intermediate solution (function value at the point $x_{0}$, tangent value of tangent line angle slope at the point $\left.x_{0}\right)$ and then the answer (tangent equation) are introduced. In total, 9 errors can be made.

Example 6. Find the normal line equation to the graph of the function $y=f(x)$ at the point $x_{0}$.

An example condition (function $f(x)$, point $x_{0}$ ), results of the intermediate solution (function value at the point $x_{0}$, tangent value of normal line angle slope at the point $\left.x_{0}\right)$ and then the answer (normal line equation) are introduced. In total, 9 errors can be made.

Example 7. Calculate extremum of the function $y=f(x)$.

An example condition (function $f(x)$ ), results of the intermediate solution (derivative of the function, critical points) are introduced and then the function is examined for the presence of an extremum at critical points.

In total, 10 errors can be made.

Example 8. Calculate the largest and smallest values of the function $y=f(x)$ on the segment $[a, b]$.

An example condition (function $f(x)$, beginning of segment $a$, end of segment $b$ ), results of an intermediate solution (expression of the derivative function, critical points, values of the function at the ends of the segment $[a, b]$ ) and then the answer (lowest value, highest value) are introduced.

In total, 12 errors can be made.

Example 9. Determine the inflection point of the graph of the function $y=f(x)$.

An example condition (function $f(x)$ ), results of an intermediate solution (expressions of derivatives of the function of the first and second orders, critical points of the second order) are introduced and then the function is examined for the presence of inflection at critical points.

In total, 12 errors can be made.

Example 10. Expand the function $y=f(x)$ into Taylor series at the point $x_{0}$ (up to the 3rd order of smallness inclusive).

An example condition (function $f(x)$, point $x_{0}$ ), results of an intermediate solution (coefficients $a_{0}, a_{1}, a_{2}, a_{3}$ ), then the answer (expansion of this function into Taylor series) are introduced.

In total, 15 errors can be made.

Example 11. Expand the function $y=f(x)$ under McLoren formula up to the 3rd order of smallness.

An example condition (function $f(x)$ ), results of an intermediate solution (coefficients $a_{0}, a_{1}, a_{2}, a_{3}$ ), then the answer (expansion of this function into McLoren series) are introduced.

In total, 15 errors can be made.

Further, all errors are summarized and, based on the amount received, a score is automatically set as follows: from 0 to 17 - "excellent", from 18 to 37 - "good", from 38 to 56 - "satisfactory", from 57 or more - "unsatisfactory"

It is also possible to output the result through a printer, so that the tester can verify the compliance of the proposed and executed option, track the errors made and the errors corrected, i.e. the printout will contain all the actions, as well as an assessment of the simulator.

In Figure 1, we can see the work of the simulator and one of the options for the work performed:

Example 1. Calculate function differential $y=x \ln x-2 x+1$.

Example 2. Calculate function differential $y=\frac{x^{2}}{x^{3}+1}$ at point $x_{0}=1$.

Example 3. Calculate the second-order function differential $y=x \arcsin x+\sqrt{1-x^{2}}-3$. 
Example 4. Calculate the second-order function differential $y=4 x^{2}+6 \sqrt[3]{x}$ at the point $x_{0}=8$.

Example 5. Find the tangent equation to the graph of the function $y=\frac{x^{2}-3 x+6}{x^{2}}$ at the point $x_{0}=3$.

Example 6. Find the normal line equation to the graph of the function $y=e^{1-x}$ at the point $x_{0}=1$.

Example 7. Calculate extremum of the function $y=\frac{2}{3} x^{2} \cdot \sqrt[3]{6 x-7}$.

Example 8. Calculate the largest and smallest values of the function $y=x^{3}+6 x^{2}-15 x$ on the segment [-1, 5].

Example 9. Determine the inflection point of the graph of the function $y=x^{3}-5 x^{2}+3 x-5$.

Example 10. Expand the function $y=x^{3}+4 x^{2}-6 x-8$ into Taylor series at the point $x_{0}=-1$ (up to the 3rd order of smallness inclusive).

Example 11. Expand the function $y=\arcsin x$ under Mcloren formula up to the 3rd order of smallness.

Since the simulator uses one hidden cell, any similar number of similar types can be made up of any number, which is important. It is worth noting that the simulator work differs from simple testing in the fact that not only the answer is introduced in the process of solving the set example, but also an intermediate solution made up of the formulas used to solve each example specifically, which allows the teacher finding the reasoning, logic and level of studied material assimilation. $[8,9,10]$

\section{Methods}

We used the following methods:

1. Theoretical - analysis of scientific literature on mathematical, methodological and special aspects related to this topic;

2. Empirical - conversations, pedagogical observations, questioning of students and teachers;

3. Programming methods in the WolframLanguage language.

\section{Results and Discussion}

The use of the simulator activates the cognitive process of students, develops thinking (visual-effective, visual-overview, subject), increases effectiveness of the educational process, allows implementing such educational goals as the development of thinking (spatial, algorithmic, intuitive, creative, theoretical types of thinking), the formation of skills to make the optimal decision from several possible options, develops the ability to carry out experimental research activities, forms an information culture, develops the ability to process information, increases students' interest in learning, and, as a result, the quality of training in mathematics and related technical and economic disciplines.

Questionings and interviews show that the opinion of teachers and students about the advisability of using the simulator in the educational process on the topic "Application of the derivative function of one variable", created and used in the Mathematica computer mathematical system, is generally positive.

\section{Summary}

When teaching mathematics using the simulator considered, important didactic principles are effectively implemented aimed at enhancing the cognitive activity of students: individualization and differentiation of the learning process (for example, due to the possibility of phased progress towards the goal along lines of varying complexity degrees), monitoring with feedback - error diagnosis (stating the reasons for the student's erroneous actions and presenting relevant comments on the computer screen) based on the results of educational activity and assessment of educational activity, the implementation of self-control, self-correction, training in the learning process. Using this simulator in the educational process does not replace traditional forms of teaching, but supplements and enriches them. Proper compilation and use of the simulator will help to significantly intensify the educational process, make the process of teaching mathematics more attractive and interesting, and prepare the student for the qualified computer use. [11]

\section{Conclusions}

The ability to quickly receive timely correction of training activities in the process of working with the simulator on the topic "Application of the derivative function of one variable" created in the Mathematica computer mathematical system stimulates students to develop their skills on this topic. This leads to an acceleration of the pace of learning, frees up time, therefore, intensifies the learning process. [12]

\section{Acknowledgements}

The work is performed according to the Russian Government Program of Competitive Growth of Kazan Federal University. 


\section{References}

[1] Zaitseva Zh.I. Methods of teaching higher mathematics using new information technologies (at a technical university): Thesis ... of the Candidate of Pedagogical Sciences: 13.00.02, 13.00.08; defended at the Moscow State Educational Institution dated 27.12.2005 / Zh.I. Zaitseva; Elabuga State Pedagogical University - Elabuga, 2005. $140 \mathrm{p}$.

[2] Zaitseva Zh.I. Teaching and controlling simulator programs in the Mathematica environment // International Scientific Seminar "Nonlinear Models in Mechanics, Statistics, Field Theory and Cosmology" - GRACOS-17. International School of Mathematical Modeling in Computer Mathematics Systems - "KAZCAS-2017". International Scientific-Practical Conference- "ITON-2017"// Materials of the seminar, school and conference/ Under the general editorship of the Honored Worker of Science of the Republic of Tatarstan, Doctor of Physical and Mathematical Sciences, Professor Yu.G. Ignatiev. - Kazan: Publishing House of the Academy of Sciences of the Republic of Tatarstan, 2017. - 279 p. - P. 170-176.

[3] Zainiev R.M., Zaitseva Zh.I. The use of computer technology in the mathematical education of students in technical areas of training // Higher Education Today. - 2015. - No. 1. - P. 19-22

[4] Zaitseva Zh.I. The use of computer technologies in the mathematical education of technical students // Kazan Science. No. 3. 2016. - Kazan: Publishing House "Kazansky Izdatelskiy Dom", 2016. - 134 - P. 108-111.

[5] Zaitseva Zh.I. The simulator program for solving math tasks created in the Mathematica computer mathematical system, on the topic "Application of the derivative function of one variable" // Certificate on state registration of a computer program No. 2015612867, 2015.

[6] Zaitseva Zh.I., Kotlyar L.M., Fomenko L.B. Organization of independent work in mathematics using modern information technologies // Fundamental Research. 2004. No. 5. P. 15-18.

[7] Kapustina, T.V., Popyrin, A.V. and Savina, L.N. (2014).Statistics and Econometrics from the Point of View Methodology Mathematics.World Applied Sciences Journal31(6):pp. 11681172,doi:10.5829/idosi.wasj.2014.31.06.14346.

[8] Kapustina, T.V., Popyrin, A.V. and Savina, L.N. (2015). Computer support of interdisciplinary communication of analytic geometry and algebra. International Electronic Journal of Mathematics EducationVolume 10 (3), pp. 177187, doi:10.12973/mathedu.2015.113a.

[9] Wolfram, S. (2003). TheMathematicaBook. Fifth Edition. Mathematica Version 5. - Wolfram Media / Cambridge University Press, 2003. - 1301 p.

[10] Wolfram, S. (2009). MathematicaDocumentationCenter. Wolfram Research, Date Views 07.03 .14 reference.wolfram.com/mathematica/guide/Mathematica.html.

[11] Engelbart D., Watson R. The augmented knowledge: Workshop//Computer Networking/Ed. By R. Blanc, I. Cotton. New York: IEEE Press, 1976.

[12] Gray A., Mezzino M., Pinsky M. Ordinary Differential Equations with Mathematica.-TELOS, 1996. 